\title{
Quality of life and its measurement: important principles and guidelines
}

\author{
M. A. Verdugo,' R. L. Schalock, ${ }^{2}$ K. D. Keith ${ }^{3} \&$ R. J. Stancliffe ${ }^{4,5}$ \\ I INICO, University of Salamanca, Salamanca, Spain \\ 2 Hastings College, Hastings, NE, USA \\ 3 University of San Diego, San Diego, CA, USA \\ 4 University of Minnesota, MN, USA \\ 5 University of Sydney, Sydney, Australia
}

\begin{abstract}
Background The importance of the valid assessment of quality of life (QOL) is heightened with the increased use of the QOL construct as a basis for policies and practices in the field of intellectual disability (ID).

Method This article discusses the principles that should guide the measurement process, the major interrogatories (i.e. who, what, when, where, why, and how) of QOL measurement, issues and procedures in the cross-cultural measurement of QOL, and the current uses of QOL data.

Results Based on the above methods, the article presents a number of important guidelines regarding QOL measurement.

Conclusion From a measurement perspective the use of the QOL construct is changing. Initially it was used as a sensitizing notion, social construct, and unifying theme. Increasingly, it is being used as conceptual framework for assessing quality outcomes, a social construct that guides quality enhancement strategies, and a criterion for assessing the effectiveness of those strategies. This new role places additional emphasis on the valid assessment of one's QOL.
\end{abstract}

Correspondence: Miguel A. Verdugo, PhD, INICO, Universidad de Salamanca, 37005 Salamanca, Spain (e-mail: verdugo@usal.es).

(C) 2005 Blackwell Publishing Ltd
Keywords cross-cultural quality of life, intellectual disability, quality of life, quality of life data, quality of life measurement

\section{Overview}

The current approach to the measurement of quality of life (QOL) can be characterized by (I) its multidimensional nature involving core domains and indicators; (2) the use of methodological pluralism that includes the use of subjective and objective measures; (3) the use of multivariate research designs to evaluate the ways that personal characteristics and environmental variables relate to the person's assessed QOL; (4) the incorporation of systems perspective that captures the multiple environments impacting people at the micro, meso, and macro levels; and (5) the increasing involvement of persons with intellectual disabilities (ID) in the design and implementation of QOL-oriented assessment, research, and evaluation. The purpose of this article is to integrate what we currently know about the measurement of QOL and to suggest, based on that knowledge, a number of relevant principles and guidelines. There are four major sections to the article: (I) the principles that should guide the measurement process; (2) the major interrogatories (i.e. who, what, when, where, why, and how) of QOL measurement; (3) 
M. A. Verdugo et al. - Quality of life and its measurement

issues and procedures in the cross-cultural measurement of QOL; and (4) the current uses to date of QOL data.

There are three areas that the article does not cover in depth: the historical approaches to QOL measurement, a survey or critique of currently used QOL assessment instruments, and family-referenced QOL. Historical approaches to the measurement of QOL can be found in Schalock \& Verdugo (2002); critiques of current measurement instruments in Cummins (2005a,b), and in Schalock \& Verdugo (2002); and an in-depth discussion of family-referenced QOL in Turnbull et al. (this volume), and in Park et al. (2003) and Poston et al. (2003). The principles and guidelines discussed in the following sections are relevant whether one is focusing on measurement of personreferenced (which is the major focus of this article) or family-referenced QOL.

\section{Quality of life measurement principles}

The principles that should guide the measurement of QOL need to be considered within the context of the current interest in the concept of QOL as a sensitizing notion, social construct and unifying theme (Schalock et al. 2002). This interest has come from three primary sources. The first is a shift in focus away from the belief that scientific, medical and technological advances alone would result in improved life toward an understanding that personal, family, community, and society well-being emerge from complex combinations of these advances plus values, perceptions and environmental conditions. The second reason reflects the fact that the QOL concept represents the next logical step from the normalization movement that stressed community-based services to measuring outcomes from the individual's life in the community. The third reason for the increased interest in the QOL concept and its measurement is the rise of the consumer empowerment movement and its emphasis on person-centred planning, personal and valued outcomes, and self-determination.

In 2002, an international panel of individuals working in the area of QOL and its measurement published a number of agreed upon principles regarding the measurement of QOL (Schalock et al. 2002). Those principles were subsequently evaluated by an additional group of 40 professionals working internationally in the measurement and application of the QOL concept to persons with ID on three social validation variables: desirability, feasibility and effectiveness (Brown et al. 2004). The result of this social validation study was discussed at the Sydney Roundtable on QOL in October, 2004. This discussion and focus group editing resulted in the following four QOL measurement principles:

I Measurement in QOL involves the degree to which people have life experiences that they value;

2 Measurement in QOL reflects domains that contribute to a full and interconnected life;

3 Measurement in QOL considers the context of physical, social, and cultural environments that are important to people; and

4 Measurement in QOL includes measured experiences both common to all humans and those unique to individuals.

\section{The interrogatories of quality of life measurement}

QOL measurement is based on at least three premises. First, QOL is important for all people and should be thought of in the same way for all people, including individuals with ID. Second, measuring QOL is required to understand the degree to which people experience a life of quality and personal wellbeing. Third, measuring QOL reflects the blend of two meanings of QOL: that which is commonly understood by human beings throughout the world, and that which has become valued by individuals as they live their lives within their unique environments. Typically, we measure the former by using QOL domains that can be reliably observed and appear to be universally held (Schalock et al. 2005b). Measuring QOL, as it is understood and valued from the individual's perspective, involves assessing perceptions of personal satisfaction or happiness (Cummins I996, I998; Schalock et al. 2002; Verdugo et al. 2005). These three premises explain why QOL is a complex phenomenon to assess. The three premises also provide the context of our summary of what we currently know about the interrogatories of QOL measurement. The following material covers four interrogatories: what, how, who and where. The why and when interrogatories will be discussed later in the section on the use of QOL assessment information. 
M. A. Verdugo et al. - Quality of life and its measurement

\section{What to measure}

There is good agreement in the QOL literature that the measurement of QOL should be based on QOL domains and indicators. Each is discussed next.

\section{Quality of life domains}

Domains refer to the set of factors composing personal well-being and should be thought of as the range over which the QOL construct extends. Most QOL investigators suggest that the number of domains is less important than the recognition that any proposed QOL model must recognize the need for a multi-element framework, the realization that people know what is important to them, and that the essential characteristics of any set of domains is that they represent in aggregate the complete QOL construct.

Recent analyses (Schalock \& Verdugo 2002; Schalock 2004) of the international QOL literature found considerable agreement regarding the personreferenced core QOL domains. Based on the published work of Hughes et al. (I995), The World Health Organization (I995), Felce \& Perry (I996), Schalock (I996), Cummins (I997), Felce (I997), Gardner \& Nudler (I997), Gettings \& Bradley (I997), Renwick et al. (2000), and Ferdinand \& Smith (2003) the most frequently referenced QOL domains (in descending frequency of being reported in the literature reviewed) are: interpersonal relations, social inclusion, personal development, physical well-being, self-determination, material wellbeing, emotional well-being, rights, environment (home/residence/living situation), family, recreation and leisure, and safety/security. These domains are quite similar to those Io domains reported by Park et al. (2003) and Poston et al. (2003) regarding family-referenced QOL: advocacy, health, production, emotional well-being, physical environment, and social well-being (individual orientation); and daily family life, family interaction, financial wellbeing, and parenting (family orientation).

\section{Quality of life indicators}

These refer to QOL domain-specific perceptions, behaviours, or conditions that give an indication of the person's well-being. These indicators are the basis for assessing quality outcomes, which refer to valued personal experiences and circumstances that: (I) follow as a result or consequence of some activity, intervention or service; and (2) are measured on the basis of quality indicators. Because of their importance as the basis for QOL measurement and the multiple uses of these indicators, the following criteria should guide their selection, measurement and use (Karon \& Bernard 2002): indicator has strategic importance for maximizing well-being; indicator measurement is expected to show variation and/or potential for improvement; indicator is useful for improving outcomes; indicator can be affected by actions taken by the provider organization and staff; indicator measure is meaningful and interpretable; data collection is feasible with reasonable efforts; costs of data collection are justified by the expected improvements in service and outcomes; indicator is sensitive to cultural and linguistic differences; indicator is applicable across populations and programmes; and indicator is based on sound theory or concepts as determined by consensus in the ID field.

\section{How to measure}

Two issues involve this interrogatory: the 'how' in terms of the focus of measurement, and the 'how' related to the measurement approach one uses.

\section{Focus of measurement}

Measuring QOL can focus on either: (I) the level of satisfaction experienced by the person in reference to the QOL domains and indicators referenced above (i.e. the subjective component of QOL measurement); or (2) objective indicators of life experience and circumstances related to those domains and indicators (i.e. the objective component of QOL measurement). Satisfaction is a commonly used dependent measure because it has a number of advantages including (Cummins 1996, I998): (I) an extensive body of research on level of satisfaction across populations and service delivery recipients; and (2) it allows one to assess the relative importance of individual QOL domains and therefore assign value to the respective domains. The major disadvantages of using only satisfaction as a dependent QOL measure include: (I) the reported low correlation between subjective and objective measures of QOL; (2) its trait-like (i.e. stability over time) nature; (3) its 
M. A. Verdugo et al. - Quality of life and its measurement

tendency to provide only a global measure of perceived well-being; and (4) the lack of demonstration to date that it is a sensitive measure of good environmental design and service programmes (Schalock \& Felce 2004; Emerson et al. 2005). Thus, both subjective and objective QOL measures are needed, and their relative weighting will depend on their anticipated use. In that regard, the following guidelines have been proposed (Schalock \& Felce 2004). I If one wants to find out whether people with ID are as satisfied with life as are other population subgroups, one should assess their level of satisfaction (on the same measures) and compare. If the scores are about the same, satisfaction is normative (Myers 2000). If not, one needs to look for personal or environmental factors that might explain such differences.

2 If one wants to evaluate environmental design or service programmes in a sensitive way, one should use objective indicators of life experiences and circumstances within the QOL framework provided by the core QOL domains and indicators.

\section{Measurement approach}

A critique of current measurement approaches can be found in Cummins (2005a,b) and Schalock \& Verdugo (2002). Across the measurement approaches, Likert-type rating and attitude scales are used most frequently, followed by various forms of questionnaires. Formal guidelines are also found in reference to one's respective approach in that the indicators measured should: (I) be related to a QOL model; (2) include subjective and objective measures; (3) have demonstrated reliability and validity; (4) have a clearly articulated use; and (5) be a guide for personal, service, or policy enhancement rather than a classification of individuals, services or systems.

\section{Who should be involved}

Traditionally the quality of one's life has typically been evaluated by others (in the case of persons with ID) or on the basis of social indicators (as in the case of societal indicators such as health, social welfare, friendships, standard of living, education, public safety, housing, neighbours and leisure). However, the quality and subjective well-being revolutions and the reform movement in the field of ID have changed the way we think about who should be involved in the measurement of one's QOL. The emerging consensus is that individuals with ID should be involved directly in the measurement of their QOL, and that proxies be used only if absolutely necessary because of significant communication limitations. This change has presented significant challenges and opportunities to the field centred around two issues: using consumers as surveyors of other consumer's QOL; and the use and influence of proxies.

Use of consumers as surveyors

In the United States, Maryland's Ask Me! Project has developed and extensively evaluated (see, e.g. Schalock et al. 2000; Bonham et al. 2004) a QOL survey instrument and procedure that involves adults with ID being trained to be the surveyor of another person's QOL on those eight domains most commonly reported in the international QOL literature. Although this procedure has required modifications in question and response format, it has resulted in reliable and valid data that are currently being used for the purposes of formative feedback and continuous programme improvement (Schalock \& Bonham 2003). From this procedure and its evaluation a number of general results have emerged including: the use of adults with ID as surveyors reduces the need for proxy respondents; simplifying the language and response formats used results in a significant increase in the number of persons with a 'profound ID' label being able to respond for themselves; response analyses indicate little if any response acquiescence or response perseveration; and if one builds proxy responses into the procedure and analysis, one is able to determine the effects of the proxy's responses. Studies in the United Kingdom of people with ID as interviewers about QOL also strongly support the capacity of people with ID to be competent data collectors (e.g. Perry \& Felce 2004).

\section{Use and influence of proxies}

In most societies people speak for themselves. When measuring QOL, the first priority should be to use all available and effective methods to enable people with ID to express their own views. These methods include simplifying the wording of questions and responses, providing pictorial response alternatives, using interpreters, and utilizing alternative and aug- 
M. A. Verdugo et al. - Quality of life and its measurement

mentative communication (e.g. Schalock et al. 2000). The process should seek to measure consumers' opinions accurately by (I) framing and presenting questions so as to minimize response biases such as acquiescence, nay saying and perseveration; and (2) by evaluating response bias to ensure that responses reflect each person's genuine views. Future research needs to find additional ways to enfranchise even more consumers in our efforts to assess the subjective component of one's QOL.

Despite serious efforts to enable people to speak for themselves about QOL, communication difficulties mean that some individuals with ID are currently unable to do so. Frequently a knowledgeable proxy is then asked to respond on the person's behalf, but there is a serious question about whether information provided by proxies is a valid and accurate substitute for self-reports.

Substantial evidence is available of lack of concordance between self-reports and proxy reports, including the self-reports of people with ID (Stancliffe 2000; Cummins 2002). Although there are also examples of satisfactory agreement between people with ID and proxies (e.g. Stancliffe I999), agreement cannot be assumed. It seems reasonable to presume disagreement unless valid consumer: proxy agreement has been demonstrated for that specific QOL instrument.

Consequently, QOL researchers face a quandary in relation to individuals who cannot communicate their own views about their QOL: either ignore these individuals because they cannot self-report, or obtain data from proxies that may be biased or invalid. Stancliffe (2000) identified several possible approaches to dealing with this issue: (I) analyze only the self-report data and disregard proxy data; (2) obtain proxy data for all participants and ignore self-reports; (3) analyze self-report and proxy data separately; and (4) correct statistically for the influence of proxies.

When both self-report and proxy data are gathered, proxies are usually used only when consumers are unable to respond for themselves. Thus information source (self-report or proxy) and personal characteristics (communication skills, adaptive behaviour) are confounded if data from the two sources are combined. In circumstances where it is important to evaluate QOL for all persons, regardless of their capacity to self-report, one valid approach is to obtain proxy data about all persons, and gather self-reports wherever possible. Data from these two sources are then analyzed separately, and there is also the opportunity to test directly the degree of agreement between selfreports and proxies, so that proxy data can be interpreted correctly.

Although proxy data cannot validly be used as a substitute for self-reports, the views of significant others about the person's QOL can be important in their own right. For example, Schwartz (2005) found that smaller community residence size was significantly associated with parents' perceptions of their son or daughter's QOL. Such findings can contribute to better understanding of parents' role in decisionmaking about out-of-family home accommodation. Depending on the purpose of the research, significant others' (i.e. proxies) views about QOL can be both valid and important. What they cannot do is replace self-reports.

\section{Where one should measure}

QOL is both multidimensional and sensitive to environmental conditions. Thus, QOL measurement should extend beyond where the person lives, works, is educated, and recreates to assessing the influence of significant persons in one's life. The importance of this extension is reflected in two lines of investigation. One line of research (e.g. that reported in Schalock et al. I994; Faulkner I995; Felce 2000; Stancliffe \& Lakin 2005) indicates clearly that personal characteristics (e.g. health status and adaptive behaviour level), environmental variables (e.g. perceived social support, type of residential setting, number of household activities participated in, earnings, and integrated activities), and care/supports provider characteristics (e.g. worker stress scores and job satisfaction ratings) are significant predictors of quality outcomes. A second line of work (e.g. that reported by Bonham et al. 2004) shows clearly that the availability of transportation and other environmental variables (e.g. service characteristics: hours of residential services, employment independence, living with family) are significant predictors of quality outcomes in people with ID.

\section{Issues and procedures in cross-cultural quality of life measurement}

Measurement of QOL across cultures entails all the conceptual, practical and psychometric issues inher- 
M. A. Verdugo et al. - Quality of life and its measurement

ent in any effort to conduct a meaningful assessment of individual well-being. However, cross-cultural measurement adds additional richness and complexity, and requires special care if the tools of measurement are to be transported from one culture to another, or if meaningful comparisons are to be made between cultures. Some of the key issues here include cultural differences in sense of self (Triandis I994), perception of others (Markus \& Kitayama I99I), cultural assumptions (Keith 1996) and the translation of meanings (Brislin I993, 2000).

\section{Sense of self}

Individuals in different cultures are enculturated at least two ways: (I) perceiving themselves in terms of their relations with others, their role in society and their contribution to meeting the needs of their group (Markus \& Kitayama I99I); or (2) perceiving themselves as independent, capable decision-makers with unique personal traits (Myers I992, 2000; Triandis I994). Thus, assumptions about happiness, wellbeing or satisfaction may not be universal, and judgments of QOL based upon such assumptions may not be generalizable from one culture to another (Goode I994; Rapley 2003). For example, Keith (I996) suggested that one culture's solitude may be another's loneliness, depending on the self-construal of the individual within his or her culture; and Kitayama \& Karasawa (1997) found that self-esteem may be perceived differently in individualistic than in collectivistic cultural settings.

\section{Perception of others}

Across cultures, individuals may be perceived quite differently along a number of dimensions such as the influences to which we attribute their behaviours, the features we find attractive, and the personality traits we value (Matsumoto \& Juang 2004). These person perception differences may even extend to critical medical conditions (Keith I996), and of course may colour judgments about individual QOL when they are seen to enhance or limit the individual in important ways.

\section{Cultural assumptions}

Among cross-cultural researchers, the terms 'etic' and 'emic' are important indicators of cultural assumptions or truths that are universal (etic) or culture-bound (emic) (Matsumoto I994; Matsumoto \& Juang 2004). The assumption that a particular dimension critical to QOL in one culture is equally important in another culture, may simply be wrong. Or, the etic-emic relationship may be more subtle. For example, although nearly every culture may value intelligence (an etic), the particular aspects of intelligence (speed, deliberation, consideration of alternatives) may vary widely (an emic) (Keith I996).

Similar differences may be found in basic values and many other cultural traits that are held dear by groups around the world. Failure to recognize these differences can lead to profoundly mistaken conclusions about comparative QOL.

\section{Translation of meanings}

When researchers want to make serious efforts to collect QOL data across cultures, equivalence becomes a major barrier that must be surmounted. Although some concepts may translate well from one language to another, many do not - and it is at this juncture that special efforts become important. As Brislin (2000) has noted, a failure to translate may indicate particular cultural emics that are not known or understood in other cultural settings, or there may simply be no readily available terminology to express such concepts in the second language. Examination of equivalence in these circumstances should often begin with back translation (Matsumoto \& Juang 2004), a procedure involving the following steps (Keith I996; Brislin 2000):

I Data collection materials (e.g. a questionnaire) are carefully developed in the researcher's native language;

2 The materials are translated (by a bilingual individual or team) to the second, or 'target' language; 3 An independent bilingual individual or team translates the materials back to the original language; 4 The two versions (original and back-translated) are then empirically evaluated for equivalence (e.g. in a test-retest format, or in analysis by bilingual experts).

If the back translation produces equivalent results across the two versions of the data collection materials, the researcher can have some confidence that specific cultural meanings have been controlled, and that something akin to semantic equivalence has been achieved (Matsumoto \& Juang 2004). However, 
M. A. Verdugo et al. - Quality of life and its measurement

investigators must take care that equivalent translations do not achieve statistical reliability at the expense of meaningful content. We have seen bilingual teams agonize over the cultural connotations of a term (e.g. 'self') even when the literal translation might be reasonably straightforward (Keith I996), leading to the caution that at least some QOL concepts may truly be embedded in culture (Rapley 2003), and therefore simply not accessible to a second culture (Brislin I993; Keith I996).

\section{Final notes on cross-cultural method}

Matsumoto \& Juang (2004, pp. 30-36) point out that the most common type of cross-cultural study involves cross-cultural comparison. However, they go on to point out that investigators should take account of several additional aspects of cross-cultural analysis. Among these are: (I) studies should look beyond differences to variables explaining why differences occur; (2) researchers should recognize the difference between ecological-level (national or cultural) studies and individual-level studies (a finding at the ecological level may not be true for every individual); (3) despite the limitations noted above, investigation of equivalence (to validate an instrument or a perspective) may sometimes be useful; and (4) sampling should often be improved in cross-cultural work (e.g. convenience samples often result in comparisons of people from different cultures who also differ markedly on other variables - urban/rural, socio-economic status, or other non-cultural demographics, among others).

In sum, cross-cultural measurement of QOL is rewarding; potentially profoundly important, particularly if it can lead to moving forward the agenda of universal improvement of QOL; and infinitely interesting if it can move us toward improved knowledge and understanding of our cultural similarities and differences. It is, nevertheless, also fraught with potential challenges that must be taken seriously if crosscultural data are to contribute to realizing the potential of meaningful application of the QOL concept.

\section{Use of quality of life data}

Studies into QOL in different scientific fields generally share a practical purpose as they look for impli- cations and personal and social changes of different nature. A recent review (Rapley 2003) of the scientific literature on QOL in different disciplines (psychology, medicine, sociology, epidemiology or disability among others) has described 23 different purposes of the research that focus in different system levels, something that was also observed through a crosscultural perspective by Keith \& Schalock (2000). The purposes established by different researchers range from comprehension, planning and evaluation of the public policies; or the coordination, training and orientation of the service providers and professionals, to the attention paid to the improvement of the consumers' active role in the habilitation and rehabilitation processes.

In the field of ID, QOL measures, based on quality indicators and frequently referred to as quality outcomes or performance measures (Schalock et al. 2005a), are typically used for four purposes, as: a measure of personal reactions, a basis for decisionmaking, a framework for programme evaluation, and a theoretical model for research. Each use to date has relied in either subjective or objective measures and has addressed a number of methodological issues involved in their use. Chief among these issues are those discussed earlier in the article: language challenges, use of proxies, psychometrics (reliability and/ or validity), and observation and reporting periods. Two general conclusions can be drawn from these four uses (Karon \& Bernard 2002; Schalock 2004): (I) as one employs quality indicators for the purposes of programme evaluation and decision-making, the use of objective measures is essential; and (2) the indicators used should meet those criteria listed earlier.

\section{Research designs}

Different research purposes involve different research questions, which in turn may need different methodologies to be answered. Quantitative approaches have been traditionally used for QOL research, but the recent increase in qualitative approaches is remarkable (Schalock \& Verdugo 2002; Verdugo \& Sabeh 2002; Rapley 2003) and must be regarded in the design of future studies.

One of the main difficulties found in measuring QOL consists in defining the sort of comparisons to be made, either within the person (e.g. examining the 
M. A. Verdugo et al. - Quality of life and its measurement

changes over time) or comparing groups. In recent years, the dominant trend has been from a 'between groups' research design to a 'multivariate/between' research design (Schalock \& Felce 2004). The use of grouped data should therefore be reduced, given that the standard of comparison is the person because measurement of QOL should be a guide for personal, service or policy enhancement rather than a classification of individuals, services or systems (Schalock \& Verdugo 2002). The use of multivariate research has many advantages. Schalock \& Felce (2004) point out some of them: focusing more on the correlates of a life of quality rather than comparing scores or status; determining the relationship among predictor variables and one's subjectively or objectively assessed QOL; and making programmatic changes and implementing techniques to enhance a person's well-being.

\section{Why measure quality of life?}

As described earlier, researches from different disciplines apply various purposes and intentions for measuring QOL. Those of us who are dedicated to the field of ID have decided to focus our attention and efforts on this concept for two reasons. First, it displays an integral and multidimensional view of the person's life that allows us to identify and plan his/ her support needs without reductionism (i.e. only focusing either on the academic curriculum, on work productivity, or on physical health). The decisions made about a person's life cannot be restricted to a limited understanding of his/her needs based on the service, organization, or programme objectives. The second reason is related to the aforementioned: discussing QOL is useful for the reorientation of the activities carried out by public organizations, service providers and professionals, giving the person an essential role as a service consumer whose perception and experience must always be taken into account. QOL and innovation complement each other.

The use of QOL data in current services and supports provides feedback about one's status on different life circumstances and events. It also allows practitioners and service providers to gather information about predictors of quality outcomes, and/or to provide data about quality outcomes and their predictors for Continuous Program Improvement (CPI). Innovation and change in programmes and services for persons with ID can be undertaken in a compre- hensive way by incorporating into person-centred planning the concepts of QOL, quality management and ethics (Spanish Confederation of Organizations in Favour of Persons with Intellectual Disability 2004). These concepts answer the main questions about what to do (improve QOL), the best way to successfully do it (quality management), and why make these efforts (ethics).

It is important to point out to researchers and professionals that the use of QOL data has not always been helpful for the purposes mentioned above. For example, when a one-dimensional (typically physical well-being) perspective is used as a singular criterion for a life of quality, judgments made over one's life have occasionally been used to make life-threatening decisions that raise numerous ethical problems (Schalock \& Verdugo 2002; Rapley 2003).

\section{When to measure}

When to measure QOL depends on one's intended use. If the aim is to reach positive, practical involvement in the life of persons with ID, long-term changes will tell us if we are on the right path. We should measure QOL at different times and compare the advances made because of the changes that have been introduced in the supports, programmes and/or services. Professionals and service providers can decide the right moment according to the suggested objectives, which may be focused either in using QOL data for CPI and/or in evaluating the change in organizations related to their CPI activities.

\section{Conclusion}

The current approach to the measurement of QOL can be characterized by its multidimensional nature involving core domains and indicators, the use of methodological pluralism that includes use of subjective and objective measures, the use of multivariate research designs to evaluate the ways that personal characteristics and environmental variables related to the person's assessed QOL, the incorporation of the systems perspective that captures the multiple environments impacting people at the micro, meso, and macrosystems levels, and the increasing involvement of persons with ID and their families in the design and implementation of QOL-oriented research and 
M. A. Verdugo et al. - Quality of life and its measurement

evaluation. In summarizing what we currently know about QOL measurement, the following four general guidelines should provide the framework for anyone interested in assessing either the subjective or objective QOL indicators: (I) QOL is both a multidimensional construct that has both subjective and objective components; (2) use the person with ID or the family (in family-centred QOL assessment) as the primary respondent; (3) use methodological pluralism (qualitative and quantitative methods) in QOL measurement; (4) be sensitive to cultural differences; and (5) base organizational and systems change on objective QOL indicators.

From a measurement perspective, the use of the QOL construct is changing. Initially, it was used as a sensitizing notion, a social construct, and a unifying theme. Increasingly, we have seen it used as a conceptual framework for assessing quality outcomes, a social construct that guides quality enhancement strategies, and a criterion for assessing the effectiveness of those strategies. This emerging use underscores the important principles and guidelines discussed in this article.

\section{References}

Bonham G. S., Basehart S., Schalock R. L., Marchand C. B., Kirchner N. \& Rumenap J. M. (2004) Consumerbased quality of life assessment: The Maryland Ask Me! Project. Mental Retardation 42, 338-55.

Brislin R. (1993) Understanding Culture's Influence on Behavior. Harcourt Brace Jovanovich, Fort Worth.

Brislin R. (2000) Understanding Culture's Influence on Behavior, 2nd edn. Harcourt College Publishers, Fort Worth.

Brown I., Keith K. D. \& Schalock R. L. (2004) Quality of life conceptualization, measurement, and application: validation of the SIRG-QOL consensus principles. Fournal of Intellectual Disability Research 48, $45 \mathrm{I}$.

Cummins R. A. (1996) The domains of life satisfaction: an attempt to order chaos. Social Indicators Research 38, 30328.

Cummins R. A. (1997) Assessing quality of life. In: Assessing Quality of Life in People with Disabilities: Models, Research, and Practices (ed. R. I. Brown), pp. II6-50. Stanley Thornes Publishers Ltd., London.

Cummins R. A. (I998) The second approximation to an international standard for life satisfaction. Social Indicators Research 43, 307-34.

Cummins R. A. (2002) Proxy responding for subjective well-being: a review. International Review of Research in Mental Retardation 25, I83-207.
Cummins R. A. (2005a) Instruments assessing quality of life: characteristics and functions. In: Approaches to the Assessment of Adults with Intellectual Disabilities: Part I: A Service Provider's Guide (eds J. H. Hogg \& A. Langa). Blackwell, London (in press).

Cummins R. A. (2005b) Issues in the systematic assessment of quality of life. In: Approaches to the Assessment of Adults with Intellectual Disabilities: Part II: Assessment Instruments: Characteristics and Functions (eds J. H. Hogg \& A. Langa). Blackwell, London (in press).

Emerson E., Robertson J., Hatton C., Knapp M., Walsh P. N. \& Hallam A. (2005) Costs and outcomes of community residential supports in England. In: Costs and Outcomes of Community Services for People with Intellectual Disabilities (eds R. J. Stancliffe \& K. C. Lakin), pp. I5 I74. Paul H. Brookes Publishers Co., Baltimore.

Faulkner E. H. (1995) Quality of life: a comparative study of institutional and community-based care for adults with mental retardation. Unpublished Doctoral Dissertation. The Graduate College in the University of Nebraska, Lincoln, NE.

Felce D. (1997) Defining and applying the concept of quality of life. Fournal of Intellectual Disability Research 41, I2635 .

Felce D. (2000) Engagement in activity as an indicator of quality of life in British research. In: Cross-cultural Perspectives on Quality of Life (eds K. D. Keith \& R. L. Schalock), pp. I73-90. American Association on Mental Retardation, Washington, DC.

Felce D. \& Perry J. (1996) Assessment of quality of life. In: Quality of Life, Volume I: Conceptualization and Measurement (ed. R. L. Schalock), pp. 63-73. American Association on Mental Retardation, Washington, DC.

Ferdinand R. \& Smith M. A. (2003) 2003 Nebraska Developmental Disabilities Provider Profiles. The ARC of Nebraska, Lincoln.

Gardner J. F. \& Nudler S. (I997) Beyond compliance to responsiveness: accreditation reconsidered. In: Quality of Life, Volume II: Application to Persons with Disabilities (ed. R. L. Schalock), pp. 135-48. American Association on Mental Retardation, Washington, DC.

Gettings R. M. \& Bradley V. J. (1997) Core Indicators Project. National Association of State Directors of Developmental Disabilities Services, Inc., Alexandria, VA.

Goode D. A. (ed.) (1994) Quality of Life for Persons with Disabilities: International Perspectives and Issues. Brookline Books, Cambridge.

Hughes C., Hwang B., Kim J., Eisenman L. T. \& Killian D. J. (I995) Quality of life in applied research: a review and analysis of empirical measures. American fournal on Mental Retardation 99, 623-4I.

Karon S. L. \& Bernard S. (2002) Development of Operational Definitions of Quality Indicators for Medicaid Services to People with Developmental Disabilities. Centers for Medicare and Medical Services, Washington, DC. 
M. A. Verdugo et al. - Quality of life and its measurement

Keith K. D. (1996) Measuring quality of life across cultures: issues and challenges. In: Quality of Life, Volume I: Conceptualization and measurement (ed. R. L. Schalock), pp. 73-82. American Association on Mental Retardation, Washington, DC.

Keith K. D. \& Schalock R. L. (eds) (2000) Cross-cultural Perspectives on Quality of Life. American Association on Mental Retardation, Washington, DC.

Kitayama S. \& Karasawa M. (1997) Implicit self-esteem in Japan: name letters and birthday numbers. Personality and Social Psychology Bulletin 23, 736-42.

Markus H. \& Kitayama S. (I99I) Culture and the self: implications for cognition, emotion, and motivation. Psychological Review 98, 224-53.

Matsumoto D. (1994) People: Psychology from a Cultural Perspective. Brooks/Cole, Pacific Grove, CA.

Matsumoto D. \& Juang L. (2004) Culture and Psychology, 3rd edn. Wadsworth, Belmont, CA.

Myers D. G. (1992) The Pursuit of Happiness: Who Is Happy and Why. William Morrow, New York.

Myers D. G. (2000) The funds, friends, and faith of happy people. American Psychologist 55, 56-67.

Park J., Hoffman L., Marquis J., Turnbull A. P., Poston D., Mannan H., Wang M. \& Nelson L. L. (2003) Toward assessing family outcomes of service delivery: validation of a family quality of life survey. Fournal of Intellectual Disability Research 47, 367-84.

Perry J. \& Felce D. (2004) Initial findings on the involvement of people with an intellectual disability in interviewing their peers about quality of life. Fournal of Intellectual and Developmental Disability 29, 164-7I.

Poston D., Turnbull A. P., Park J., Mannan H., Marquis J. \& Wang M. (2003) Family quality of life: a qualitative inquiry. Mental Retardation 4I, 313-28.

Rapley M. (2003) Quality of Life Research: a Critical Introduction. SAGE, London.

Renwick R., Brown I. \& Raphael D. (2000) Person-centered quality of life: contributions from Canada to an international understanding. In: Cross-cultural Perspectives on Quality of Life (eds K. R. Keith \& R. L. Schalock), pp. 5-23. American Association on Mental Retardation, Washington, DC.

Schalock R. L. (I996) Reconsidering the conceptualization and measurement of quality of life. In: Quality of Life, Volume I: Conceptualization and Measurement (ed. R. L. Schalock), pp. I23-39. American Association on Mental Retardation, Washington, DC.

Schalock R. L. (2004) Quality of life: what we know and do not know. Fournal of Intellectual Disability Research 48, 203-I6.

Schalock R. L. \& Bonham G. S. (2003) Measuring outcomes and managing for results. Evaluation and Program Planning 26, 229-35.
Schalock R. L., Bonham G. S. \& Marchand C. B. (2000) Consumer based quality of life assessment: a path model of perceived satisfaction. Evaluation and Program Planning 23, 77-88.

Schalock R. L., Bradley V. J. \& Gardner J. F. (2005a) Quality Outcomes for Persons with Disabilities: a Comprehensive Guide to Performance Enhancement. American Association on Mental Retardation, Washington, DC (in press).

Schalock R. L., Brown I., Brown R., Cummins R. A., Felce D., Matikka L., Keith K. D. \& Parmenter T. (2002) Conceptualization, measurement, and application of quality of life for persons with intellectual disabilities: results of an international panel of experts. Mental Retardation 40, 457-70.

Schalock R. L. \& Felce D. (2004) Quality of life and subjective well-being: conceptual and measurement issues. In: International Handbook on Methods for Research and Evaluation in Intellectual Disabilities (eds E. Emerson, T. Thompson, T. Parmenter \& C. Hatton), pp. 26I-80. Wiley, New York.

Schalock R. L., Lemanowicz J. A., Conley J. W. \& Feinstein C. S. (1994) A multivariate investigative study of the correlates of quality of life. Fournal on Developmental Disabilities 3, 59-73.

Schalock R. L. \& Verdugo M. A. (2002) Handbook on Quality of Life for Human Service Practitioners. American Association on Mental Retardation, Washington, DC.

Schalock R. L., Verdugo M. A., Jenaro C., Wang M., Wehmeyer M., Xu J. \& Lachapelle Y. (2005b) A cross-cultural study of quality of life indicators. American fournal on Mental Retardation II0, 298-3II.

Schwartz C. (2005) Parental involvement in residential care and perceptions of their offspring's life satisfaction in residential facilities for adults with intellectual disability. fournal of Intellectual and Developmental Disability (in press).

Spanish Confederation of Organizations in Favour of Persons with Intellectual Disability (2004) Cartera de Servicios Sociales Del Movimiento FEAPS [Social services portfolio of the FEAPS movement]. Author, Madrid.

Stancliffe R. J. (1999) Proxy respondents and the quality of life questionnaire empowerment factor. Fournal of Intellectual Disability Research 43, 185-93.

Stancliffe R. J. (2000) Proxy respondents and quality of life. Evaluation and Program Planning 23, 89-93.

Stancliffe R. J. \& Lakin K. C. (2005) Costs and Outcomes of Community Services for People with Intellectual Disabilities. Paul H. Brookes Publishers Co., Baltimore.

The World Health Organization Quality of Life Group (1995) The World Health Organization Quality of Life Assessment (WHOQOL): position paper from the World Health Organization. Social Science Medicine 42, I403-9. 
M. A. Verdugo et al. - Quality of life and its measurement

Triandis H. C. (1994) Culture and social behavior. In: Psychology and Culture (eds W. J. Lonner \& R. Malpass), pp. I69-73. Allyn \& Bacon, Boston.

Verdugo M. A., Prieto G., Caballo C. \& Pelaez A. (2005) Factorial structure of the quality of life questionnaire in a Spanish sample of visually disabled adults. European fournal of Psychological Assessment 21, 44-55.
Verdugo M. A. \& Sabeh E. N. (2002) Evaluación de la percepción de calidad de vida en la infancia. [Perceived quality of life assessment in childhood]. Psicothema $\mathbf{1 4}$, 86-9I. 